\title{
Acoustic eigenanalysis for multiply-connected problems using dual BEM
}

\author{
J. T. Chen ${ }^{1, * \dagger}$, L. W. Liu ${ }^{1}$ and S. W. Chyuan ${ }^{2}$ \\ ${ }^{1}$ Department of Harbor and River Engineering, National Taiwan Ocean University, Keelung, Taiwan \\ ${ }^{2}$ Chungshan Institute of Science and Technology, Lungtan, Taiwan
}

\begin{abstract}
SUMMARY
In this paper, the eigenanalysis for the multiply-connected domain problem is studied by using the dual boundary element method. The occurrence and treatment of the spurious eigenvalues for multiplyconnected domain problem are reviewed when the complex-valued BEM in used. Three approaches, Burton and Miller method, CHIEF concept and SVD updating techniques, are adopted to suppress the occurrence of spurious eigensolutions. Instead of using the singular and hypersingular formulations, the singularity-free methods, the null-field equation approach and the fictitious BEM, are also utilized to deal with the eigenproblem. Both the eigenvalues and eigenmodes are compared with the analytical solutions and those of FEM for the illustrative examples. Good agreement is made. Copyright (c) 2004 John Wiley \& Sons, Ltd.
\end{abstract}

KEY WORDS: boundary element method; multiply-connected domain; singular value decomposition; Fredholm alternative theorem; CHIEF concept

\section{INTRODUCTION}

Boundary element method (BEM) has been accepted as an alternative for solving the acoustic eigenproblem. For simply-connected domain problems, the dual reciprocity method (DRM) [1] and the multiple reciprocity method (MRM) [2] have been widely used. Both the aforementioned methods belong to real-valued formulations. One advantage of the MRM, which uses the Laplace-type fundamental solution, is that only real-valued computation is needed [3]. Therefore, the MRM is indeed no more than the real part of the complex-valued formulation [4]. Tai and Shaw [5] and De Mey [6,7] employed a simplified method of using either the real- or the imaginary-part kernels. Hutchinson [8] also employed the real-part

\footnotetext{
*Correspondence to: J. T. Chen, Department of Harbor and River Engineering, National Taiwan Ocean University, P.O. Box 7-59, Keelung, Taiwan, Republic of China.

†E-mail: jtchen@mail.ntou.edu.tw

Contract/grant sponsor: National Science Council; contract/grant number: NSC-91-2211-E-019-009
}

Copyright (c) 2004 John Wiley \& Sons, Ltd.

Received 17 October 2002 Accepted 5 September 2003 
kernels to solve membrane vibration problems. However, both real-part singular and hypersingular equations, yield spurious eigenvalues. The occurrence of spurious eigenvalue is the major drawback of the real-part BEM for solving the acoustic eigenproblem. To deal with the problem of spurious eigenvalue, the dual MRM [9], the real-part dual BEM [10], the singular value decomposition (SVD) updating terms and updating documents [11,12] and the generalized singular value decomposition (GSVD) [13] have been constructed. In addition, Chen et al. [14] extended the CHIEF concept $[15,16]$ to the combined Helmholtz exterior integral equation formulation (CHEEF) method for filtering out the spurious eigenvalues. In fact, there are no spurious eigenvalues if the complex-valued BEM is employed for a simply-connected problem as Tai and Shaw [5] pointed out. However, spurious eigensolutions also appear for multiply-connected problems even when the complex-valued BEM is employed [17, 18]. In Chen et al. [17], the problem of spurious eigensolutions encountered in the singular and hypersingular BEMs was studied by using circulants for an annular case and was treated by using the Burton and Miller approach [19]. The continuous formulation was also studied by Chen et al. [20].

Rigorously speaking, the aforementioned domain of interest [17,20] is doubly connected instead of multiply connected. However, the research conducted prior to this investigation did not address both the occurring mechanism of spurious eigenvalues and the detection of the spurious eigenvalues for the truly multiply-connected problem. To solve the multiplyconnected eigenproblem, Lin [21] employed the transformation technique of cylindrical wave functions to satisfy the boundary condition for finding the eigenvalues of an eccentric annular domain and a circular domain with seven equal holes. Nagaya and Poltorak [22] used the point-matching approach to find the eigenvalues of a circular domain with eccentric circular inner boundaries. Nagaya and Yamaguchi [23] used both the Fourier expansion collocation method and point-matching approach to find the eigenvalues of the elliptical or polygonal outer boundary with eccentric inner boundaries. However, all those approaches were not compared with other numerical approaches, e.g. FEM or BEM, even though the exact solutions were not available.

In this paper, we will employ the boundary element method to determine the eigenvalue and eigenmode for the multiply-connected eigenproblem. The methods of filtering out the spurious eigenvalue by using either the CHIEF method or the SVD updating techniques will be discussed for the direct and indirect BEMs. Also, the Burton and Miller approach is considered for comparison. In addition, the techniques of detecting the true eigenvalue will be addressed. Numerical experiments will be performed to verify the present formulation. The relations between the spurious eigenvalue and the associated formulation will be examined. For the multiply-connected problem, the mode shapes will be plotted by using the BEM and will be compared with the other available results, e.g. exact solution and FEM data.

\section{BOUNDARY INTEGRAL EQUATIONS FOR MULTIPLY-CONNECTED PROBLEM}

The governing equation of the acoustic problem is the Helmholtz equation

$$
\left(\nabla^{2}+k^{2}\right) u(\mathbf{x})=0, \quad \mathbf{x} \in D
$$


where $\nabla^{2}, k$ and $D$ are the Laplacian operator, the wave number, and the domain of interest, respectively. On the basis of the dual boundary integral formulation [24,25], we have

$$
\begin{aligned}
& \alpha u(\mathbf{x})=\operatorname{CPV} \int_{B} T(\mathbf{s}, \mathbf{x}) u(\mathbf{s}) \mathrm{d} B(\mathbf{s})-\operatorname{RPV} \int_{B} U(\mathbf{s}, \mathbf{x}) t(\mathbf{s}) \mathrm{d} B(\mathbf{s}), \quad \mathbf{x} \in B \\
& \alpha t(\mathbf{x})=\operatorname{HPV} \int_{B} M(\mathbf{s}, \mathbf{x}) u(\mathbf{s}) \mathrm{d} B(\mathbf{s})-\operatorname{CPV} \int_{B} L(\mathbf{s}, \mathbf{x}) t(\mathbf{s}) \mathrm{d} B(\mathbf{s}), \quad \mathbf{x} \in B
\end{aligned}
$$

where $\mathbf{x}$ is the boundary point, $\mathbf{s}$ is the source point, $B$ is the boundary, RPV denotes the Reimann principal value, CPV denotes the Cauchy principal value, HPV denotes the Hadamard principal value, $t(\mathbf{s})$ is the directional derivative of $u(\mathbf{s})$ along the outer normal direction at $\mathbf{s}$, and $\alpha$ is the interior angle of the boundary at $\mathbf{x}$. The $U(\mathbf{s}, \mathbf{x}), T(\mathbf{s}, \mathbf{x}), L(\mathbf{s}, \mathbf{x})$ and $M(\mathbf{s}, \mathbf{x})$ represent the four kernel functions [23]. Equation (2) is referred to as the singular BIE and Equation (3) the hypersingular BIE. The combined use of both is termed the dual BIEs. Corresponding to Equations (2) and (3), the null-field BIEs [26] based on the direct method are

$$
\begin{array}{ll}
0=\int_{B} T(\mathbf{s}, \mathbf{x}) u(\mathbf{s}) \mathrm{d} B(\mathbf{s})-\int_{B} U(\mathbf{s}, \mathbf{x}) t(\mathbf{s}) \mathrm{d} B(\mathbf{s}), & \mathbf{x} \in D^{e} \\
0=\int_{B} M(\mathbf{s}, \mathbf{x}) u(\mathbf{s}) \mathrm{d} B(\mathbf{s})-\int_{B} L(\mathbf{s}, \mathbf{x}) t(\mathbf{s}) \mathrm{d} B(\mathbf{s}), & \mathbf{x} \in D^{e}
\end{array}
$$

where $D^{e}$ is the complementary domain and the kernels are the same as listed in Reference [24]. Note that the null-field BIEs are not singular. The kernel functions in the null-field BIEs can be expanded into the degenerate kernels [20]. In order to avoid singularity, the fictitious boundary formulation is another choice as well as the null-field formulations. Here, we present the fictitious BIEs adopting the single- and double-layer potential approaches of indirect method. For the single-layer potential approach, the single-layer density $\phi$ is distributed on the fictitious boundary $B^{\prime}$ and the field solutions are represented in terms of the single-layer potential,

$$
\begin{aligned}
& u(\mathbf{x})=\int_{B^{\prime}} U(\mathbf{s}, \mathbf{x}) \phi(\mathbf{s}) \mathrm{d} B(\mathbf{s}) \\
& t(\mathbf{x})=\int_{B^{\prime}} L(\mathbf{s}, \mathbf{x}) \phi(\mathbf{s}) \mathrm{d} B(\mathbf{s})
\end{aligned}
$$

For the double-layer potential approach of the indirect method,

$$
\begin{aligned}
& u(\mathbf{x})=\int_{B^{\prime}} T(\mathbf{s}, \mathbf{x}) \psi(\mathbf{s}) \mathrm{d} B(\mathbf{s}) \\
& t(\mathbf{x})=\int_{B^{\prime}} M(\mathbf{s}, \mathbf{x}) \psi(\mathbf{s}) \mathrm{d} B(\mathbf{s})
\end{aligned}
$$

where the double-layer density $\psi$ is distributed on the fictitious boundary $B^{\prime}$. 


\section{TREATMENTS OF SPURIOUS EIGENVALUES}

Following the developed techniques $[17,20]$, the true and spurious eigensolutions will be detected and distinguished. Two concepts are addressed here. The spurious eigensolution depends on the formulation instead of the types of boundary conditions for real problems. On the other hand, the true eigensolutions (eigenvalue and eigenmode) are embedded in the formulation and are different for the Dirichlet and Neumann problems.

\subsection{Detection of spurious eigenvalues in the direct BEMs by using SVD updating documents}

According to the Fredholm alternative theorem and the concept of spurious resonance [20] in the non-homogeneous boundary condition, we find that the spurious modes $\left(\phi_{\mathrm{s}}\right)$ for the Dirichlet and Neumann problems can be identical and that

$$
\left[\begin{array}{c}
U^{\mathrm{H}}\left(k_{\mathrm{s}}\right) \\
T^{\mathrm{H}}\left(k_{\mathrm{s}}\right)
\end{array}\right]\left\{\phi_{\mathrm{s}}\right\}=\{0\}
$$

where $k_{\mathrm{s}}$ is the spurious wavenumber, and the superscript $\mathrm{H}$ denotes the Hermitian conjugate. Taking the Hermitian conjugate with respect to Equation (10), we have

$$
\left\{\phi_{\mathrm{s}}\right\}^{\mathrm{H}}\left[U\left(k_{\mathrm{s}}\right) T\left(k_{\mathrm{s}}\right)\right]=\{0\}^{\mathrm{H}}
$$

From the preceding argument, the two matrices $[U]$ and $[T]$ have the same spurious mode $\left\{\phi_{\mathrm{s}}\right\}$ corresponding to the common spurious eigenvalue $k_{\mathrm{s}}$.

Similarly, the $L M$ method has the same spurious mode $\left(\bar{\phi}_{\mathrm{s}}\right)$ corresponding to each spurious eigenvalue $\bar{k}_{\mathrm{s}}$ for the Dirichlet and Neumann problems as shown below:

$$
\begin{gathered}
{\left[\begin{array}{c}
L^{\mathrm{H}}\left(\bar{k}_{\mathrm{s}}\right) \\
M^{\mathrm{H}}\left(\bar{k}_{\mathrm{s}}\right)
\end{array}\right]\left\{\bar{\phi}_{\mathrm{s}}\right\}=\{0\}} \\
\left\{\bar{\phi}_{\mathrm{s}}\right\}^{\mathrm{H}}\left[L\left(\bar{k}_{\mathrm{s}}\right) M\left(\bar{k}_{\mathrm{s}}\right)\right]=\{0\}^{\mathrm{H}}
\end{gathered}
$$

By the same token as in the $U T$ method, the $[L]$ and $[M]$ matrices have the same spurious eigenvalues. In other words, the spurious eigenvalues are related to whether formulated by the $U T$ method or by the $L M$ method rather than related to whether the boundary condition is of the Dirichlet type or the Neumann type.

To detect the spurious eigenvalues, we merge the $[U]$ and $[T]$ matrices to form the so-called updating document

$$
[B(k)]=[U(k) T(k)]
$$

By applying SVD technique for $[B(k)]$, the minimum singular value of $[B(k)]$ as a (numerical) function of $k$ can be utilized to find the spurious eigenvalues $k_{\mathrm{s}}$ and the spurious modes $\left\{\phi_{\mathrm{s}}\right\}$ at the same time. 


\subsection{Detection of true eigenvalues in the direct BEMs by using SVD updating terms}

Consider that the true eigensolution must be embedded in

$$
\begin{aligned}
{\left[U\left(k_{\mathrm{t}}\right)\right]\{t\} } & =\{0\} \\
{\left[L\left(k_{\mathrm{t}}\right)\right]\{t\} } & =\{0\}
\end{aligned}
$$

for the homogeneous Dirichlet problem, where $k_{\mathrm{t}}$ denotes the true wavenumber. Equation (14) indicates that both the $[U]$ and $[T]$ matrices have the same zero singular value corresponding to the right unitary vector $\{t\}$. This finding guides us to merge the two equations together,

$$
\left[D\left(k_{\mathrm{t}}\right)\right]\{t\}=\{0\}
$$

where

$$
\left[D\left(k_{\mathrm{t}}\right)\right]=\left[\begin{array}{l}
U\left(k_{\mathrm{t}}\right) \\
L\left(k_{\mathrm{t}}\right)
\end{array}\right]
$$

By plotting the minimum singular value of $[D(k)]$ versus $k$, one has a curve which drops at the positions of true eigenvalues.

The technique of SVD updating term can also be applied to the Neumann problem,

$$
[N(k)]\{u\}=\{0\}
$$

where

$$
[N(k)]=\left[\begin{array}{c}
T(k) \\
M(k)
\end{array}\right]
$$

To detect true eigenvalues, a similar procedure for the minimum singular value of matrix $[N(k)]$ versus $k$ can be developed.

\subsection{Detection of true eigenvalues in the indirect BEMs by using SVD updating documents}

According to the Fredholm alternative theorem for the non-homogeneous Dirichlet problem $(u=\bar{u}$ on $B)$ in indirect BEMs [20], we obtain

$$
\left[\begin{array}{c}
U^{\mathrm{H}}\left(k_{\mathrm{t}}\right) \\
T^{\mathrm{H}}\left(k_{\mathrm{t}}\right)
\end{array}\right]\{\eta\}=\{0\}
$$

where $\eta$ is the true eigenmode. To detect the true eigenvalues, we plot the minimum singular value of the assembled matrix $\left[\begin{array}{c}U^{\mathrm{H}}(k) \\ T^{\mathrm{H}}(k)\end{array}\right]$ versus $k$, and the curve drops indicate the positions of the true eigenvalues.

The technique of SVD updating document can be extended to the Neumann problem. The minimum singular value for the assembled matrix $\left[\begin{array}{c}L^{\mathrm{H}}(k) \\ M^{\mathrm{H}}(k)\end{array}\right]$ versus $k$ is plotted, and the drops in the curve are also found at the positions of true eigenvalues. 


\subsection{Detection of spurious eigenvalues in the indirect BEMs by using SVD updating terms}

For the homogeneous Dirichlet and Neumann eigenproblems, we have

$$
\begin{array}{r}
{\left[U\left(k_{\mathrm{s}}\right)\right]\{\varphi\}=\{0\}} \\
{\left[L\left(k_{\mathrm{s}}\right)\right]\{\varphi\}=\{0\}}
\end{array}
$$

Combining Equations (20) and (21), we have

$$
\left[\begin{array}{c}
U\left(k_{\mathrm{s}}\right) \\
L\left(k_{\mathrm{s}}\right)
\end{array}\right]\{\varphi\}=\{0\}
$$

The minimum singular value of the assembled matrix $\left[\begin{array}{l}U(k) \\ L(k)\end{array}\right]$ versus $k$ is then plotted, the local minima of the resulted curve revealing the spurious eigenvalues $k_{\mathrm{s}}$. Next let us consider

$$
\left[\begin{array}{c}
T\left(k_{\mathrm{s}}\right) \\
M\left(k_{\mathrm{s}}\right)
\end{array}\right]\{\psi\}=\{0\}
$$

for detecting the spurious eigenvalues occurring in the double-layer potential approach. The minimum singular value of the assembled matrix $\left[\begin{array}{c}T(k) \\ M(k)\end{array}\right]$ versus $k$ can be similarly examined to find the spurious eigenvalues $k_{\mathrm{s}}$.

\subsection{Extraction of true eigenvalues using CHIEF point and the Burton and Miller method}

In order to overcome the problem of the spurious eigenvalue, the CHIEF points are chosen and the overdetermined system of Dirichlet problem can be obtained [20]. Another treatment is the Burton and Miller method [17].

\section{NUMERICAL EXPERIMENTS FOR MULTIPLY-CONNECTED PROBLEMS}

In order to verify not only the occurring mechanism of spurious eigensolution but also the suppression of the spurious eigenvalues for the multiply-connected problem, numerical experiments for four examples were performed. In the literature, the inner boundaries of multiply-connected domain were designed with identical geometry. In order to understand how geometry of inner boundaries influences spurious eigenvalue; hence, we designed a case of a circular domain with two unequal inner holes. The other problems, the cases of a circular domain with two, four and seven equal holes were performed to compare with other available solutions.

\subsection{A circular domain with two unequal holes}

The circular domain with a radius $R=1 \mathrm{~m}$ and two circular inner boundaries where the eccentricity $e$ is $0.5 \mathrm{~m}$ with radii of $c_{1}=0.3 \mathrm{~m}$ and $c_{2}=0.4 \mathrm{~m}$, respectively, are considered in Table I. All the boundary conditions are the Dirichlet types $(u=0)$. By using the eight 
Table I. The former five eigenvalues for a multiply-connected problem with two unequal holes using different approaches.

\begin{tabular}{l} 
(1) \\
\hline
\end{tabular}

approaches $[17,20]$ in Table I, the former five eigenvalues $\left(k_{i}, i=1,2, \ldots, 5\right)$ are obtained as shown in Table I. The former two eigenvalues $\left(k_{1}, k_{2}\right)$ and the subsequent two $\left(k_{3}, k_{4}\right)$ are roots of multiplicity two which can be distinguished by using the BEMs + SVD updating techniques. However, FEM calculations cannot distinguish the multiplicity as shown in Table I since $k_{1} \neq k_{2}$ and $k_{3} \neq k_{4}$. Good agreement for the five eigenmodes corresponding to the former five eigenvalues by using the BEM and FEM is obtained in Figure 1 where a plus sign and a minus sign denote the convex and concave sides, respectively. Although the mode shapes corresponding to the eigenvalues $k_{3}$ and $k_{4}$ seem different between the results of BEM 

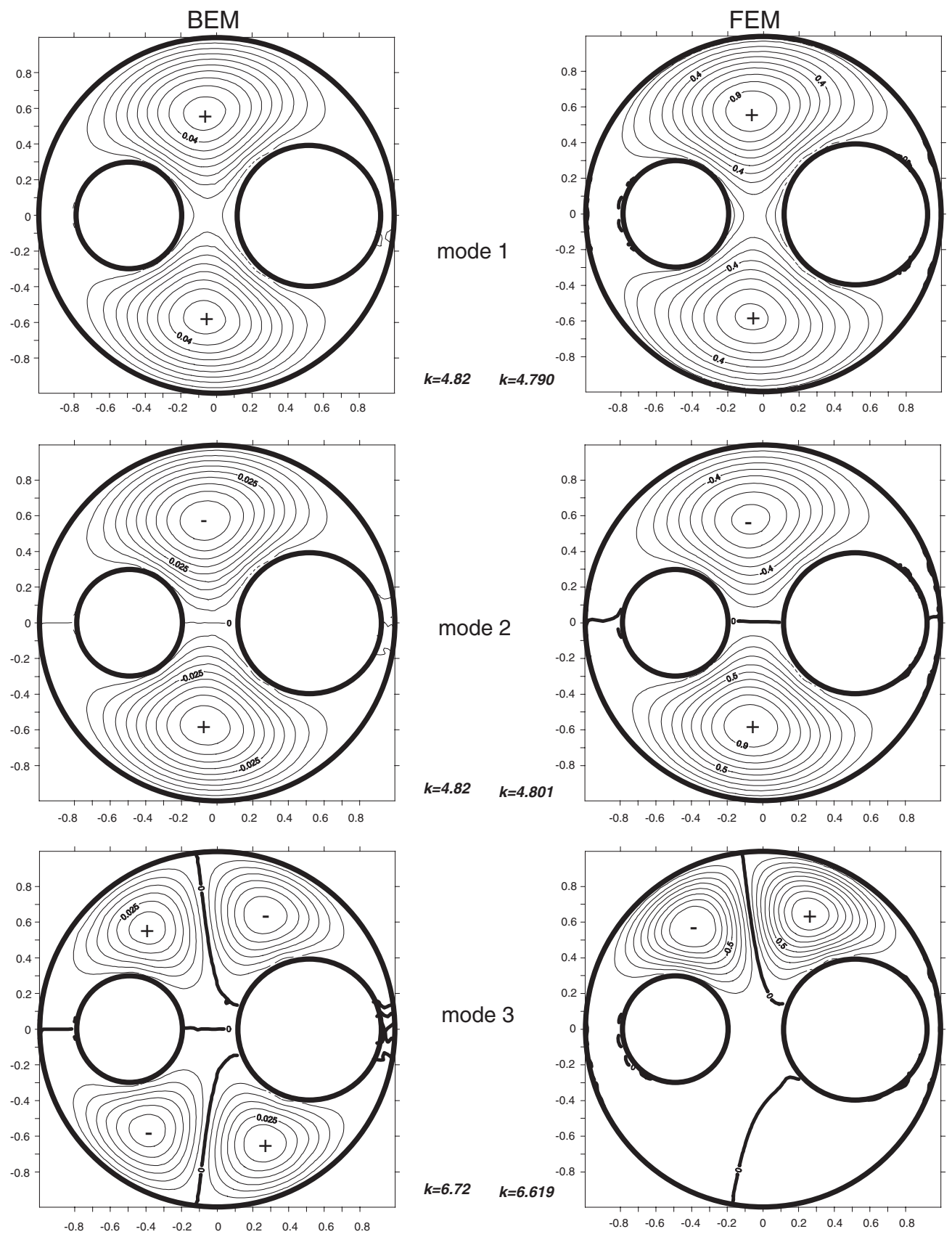

Figure 1. The former five modes for a multiply-connected problem with two unequal holes. 

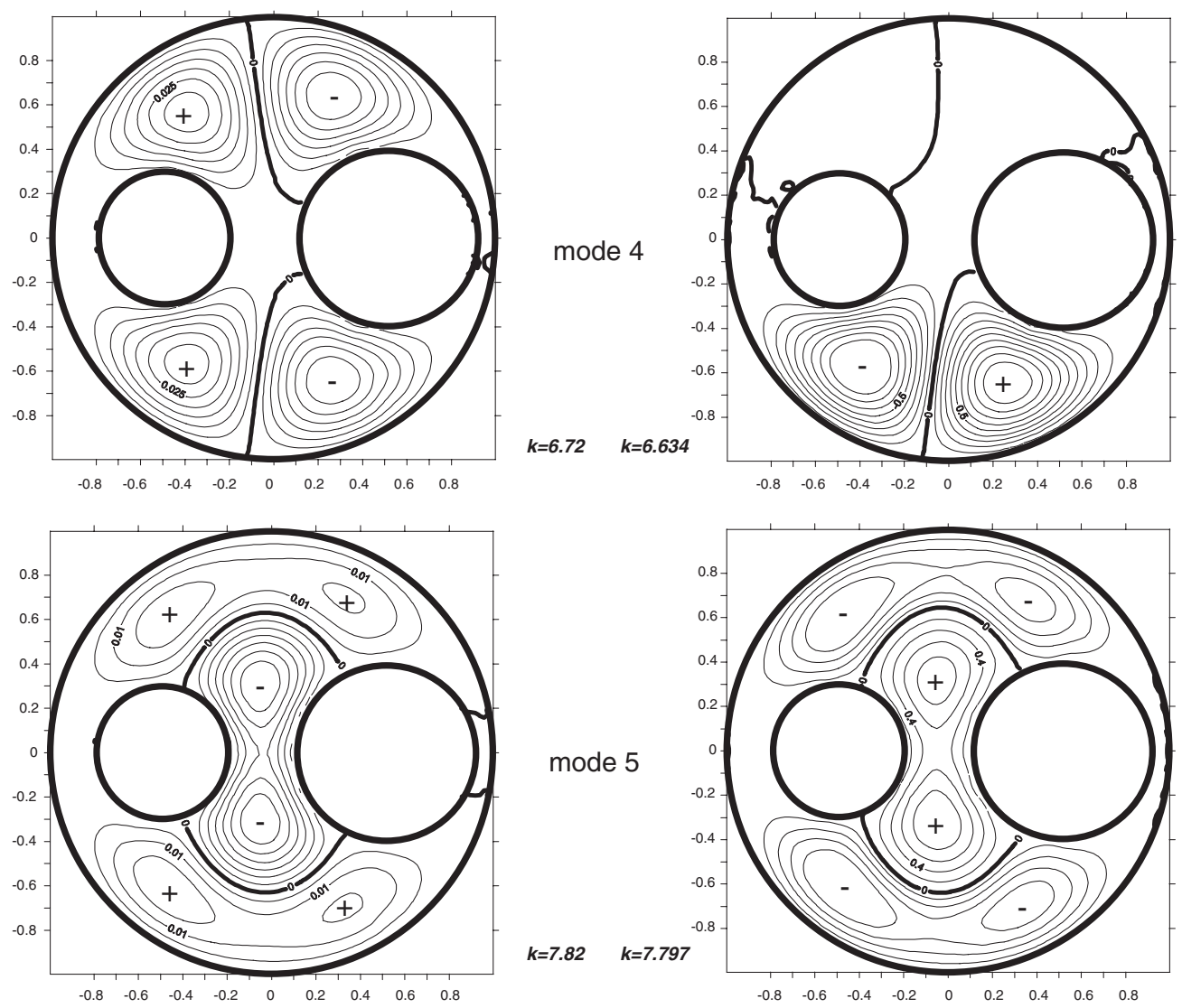

Figure 1. Continued.

and FEM, each mode shape of BEM for $k_{3}$ and $k_{4}$ can be obtained by linearly superimposing the two independent mode shapes of FEM, and vice versa. Similarly, the mode shape corresponding to the eigenvalue $k_{5}$ is different by a factor -1 between the results of BEM and FEM. Comparing the data of BEM with that of FEM, the root of multiplicity two can be detected by using BEM instead of FEM. To sort out spurious eigenvalues, the direct BEM and the SVD updating document are employed. Figure 2 shows that the spurious eigenvalues appear in the location of the $k_{\mathrm{s}}=6.16\left(J_{0}^{1} / c_{2}=6.012\right)$ or $8.20\left(J_{0}^{1} / c_{1}=8.016\right)$ in the range of $0<k \leqslant 9$, where the exact values are shown in the parentheses. It is a clear evidence to demonstrate that the spurious eigensolution depends on the radii of inner circles $\left(c_{1}\right.$ and $\left.c_{2}\right)$ as predicted by Chen et al. $[17,20]$ for the annular case.

By using 15 elements for each inner boundary and 60 elements for the outer boundary, the spurious boundary mode $\Phi_{1}$ from the left unitary matrix of SVD corresponding to the zero singular value for the spurious eigenvalue is shown in Figure 3(a)-3(d) for the four cases. To demonstrate how spurious mode relates to the spurious eigenvalue, the former four spurious eigenvalues of the $k_{\mathrm{s}}=6.16\left(J_{0}^{1} / c_{2}=6.012\right), 8.20\left(J_{0}^{1} / c_{1}=8.016\right), 9.65\left(J_{1}^{1} / c_{2}=9.579\right)$ and $13.00\left(J_{1}^{1} / c_{1}=12.772\right)$ are considered where the theoretical predictions are shown in the parentheses. It is found that the spurious boundary mode $\Phi_{1}$ is zero except on the inner boundary $c_{j}$ 


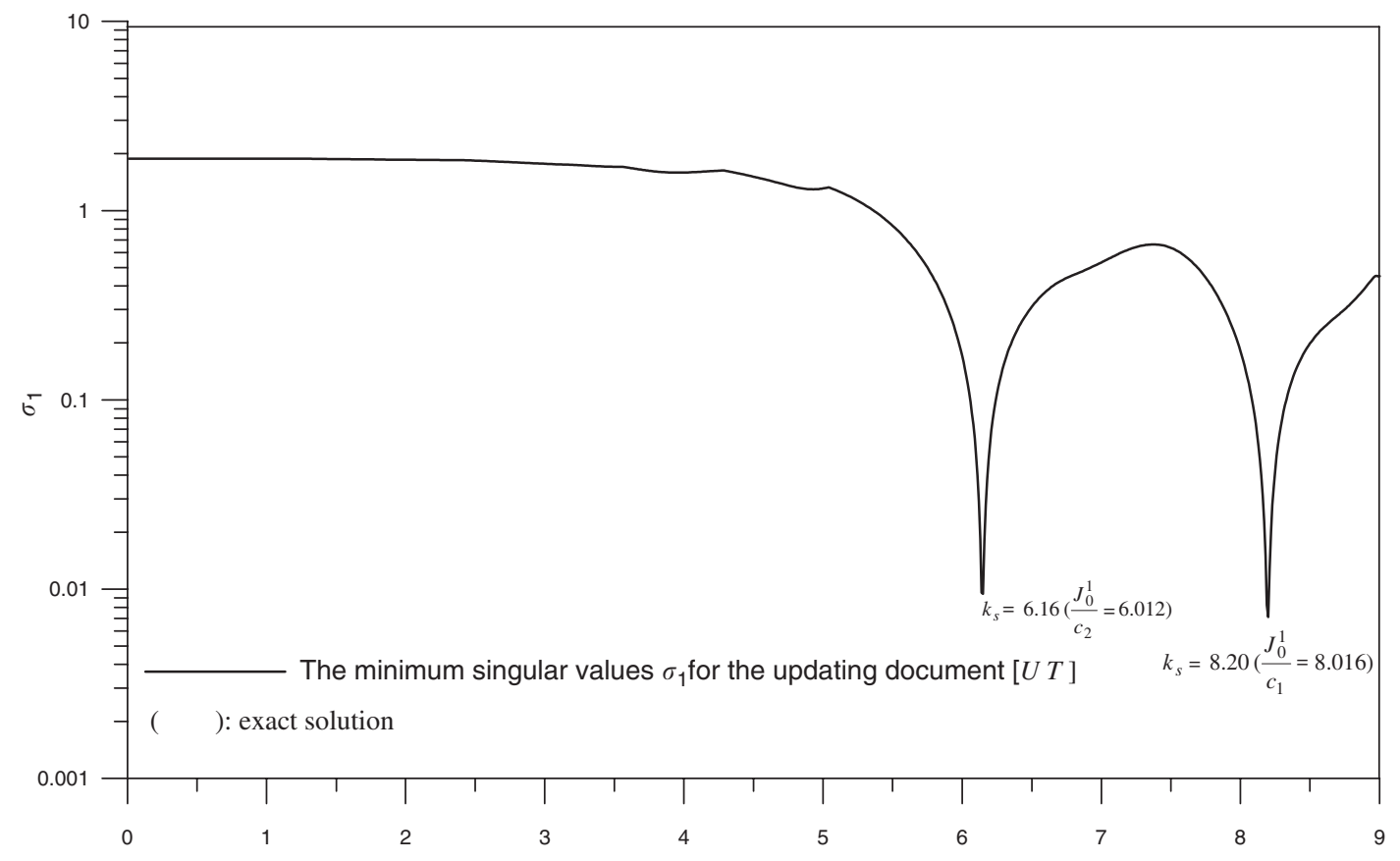

Figure 2. Detection of spurious eigenvalues using the SVD updating document.

(where $j=1,2$ ) for the corresponding spurious eigenvalue $k_{\mathrm{s}}=J_{n}^{1} / c_{j}$ (where $j=1,2, n \in \mathbf{N}$ ), respectively. In addition, the former two spurious eigenvalues satisfying $J_{0}\left(k_{\mathrm{s}} c_{i}\right)=0$ (where $i=1,2$ ) have the same spurious mode of constants $\left(\mathrm{e}^{\mathrm{i} 0 \theta}\right)$ as shown in Figure 3(a) and 3(b) while the subsequent two spurious eigenvalues satisfying $J_{1}\left(k_{\mathrm{s}} c_{j}\right)=0$ (where $j=1,2$ ) have the same spurious mode of $\sin \theta$ or $\cos \theta\left(\mathrm{e}^{\mathrm{i} 1 \theta}\right)$ as shown in Figure 3(c) and 3(d). It is theoretically predicted that the spurious eigenvalues $k_{\mathrm{s}}$ satisfying $J_{n}\left(k_{\mathrm{s}} c_{j}\right)=0$ (where $j=1,2$ ) have the same spurious mode of $\sin (n \theta)$ or $\cos (n \theta)\left(\mathrm{e}^{\mathrm{i} n \theta}\right)$. Hence, the singularity pattern resulting in a nullfield solution is found in the numerical demonstration [17, 20,27]. In addition, only two CHIEF points $(0.5,0)$, and $(-0.5,0)$ inside each circle are required to sort out the spurious eigenvalues $k_{\mathrm{s}}=6.16\left(J_{0}^{1} / c_{2}=6.012\right)$ and $k_{\mathrm{s}}=8.20\left(J_{0}^{1} / c_{1}=8.016\right)$ of multiplicity one, respectively.

\subsection{A circular domain with two equal holes}

For the problem of a circular domain with two equal holes, Nagaya and Poltorak [22] used the point-matching (PM) approach for finding the eigenvalues. The radius $R=1 \mathrm{~m}$ of the outer boundary and the radii $c=0.3 \mathrm{~m}$ of the inner boundaries with the eccentricity $e=0.5 \mathrm{~m}$ are considered in Table II. The boundary conditions along the inner and outer boundaries are the Dirichlet types $(u=0)$. Numerical data of the three approaches, point-matching method [22], BEM and FEM, are listed in the Table II. For the root of multiplicity two, the former two eigenvalues $\left(k_{1}, k_{2}\right)$ and the subsequent two $\left(k_{3}, k_{4}\right)$ are obtained in the BEM results while FEM cannot distinguish the multiplicity. Following the data of point-matching approach in the parentheses, the symmetry and antisymmetry of the mode shape are noted by Nagaya 
and Poltorak where the (S) and (A) symbols denote the symmetric and antisymmetric with respect to the $x$ - and $y$-axis, respectively. The same symbols are used in the BEM results. It is easy to find that the symmetry of mode shape of BEM and point-matching approach matched well. For the plots of the former five modes using the BEM and the FEM as shown

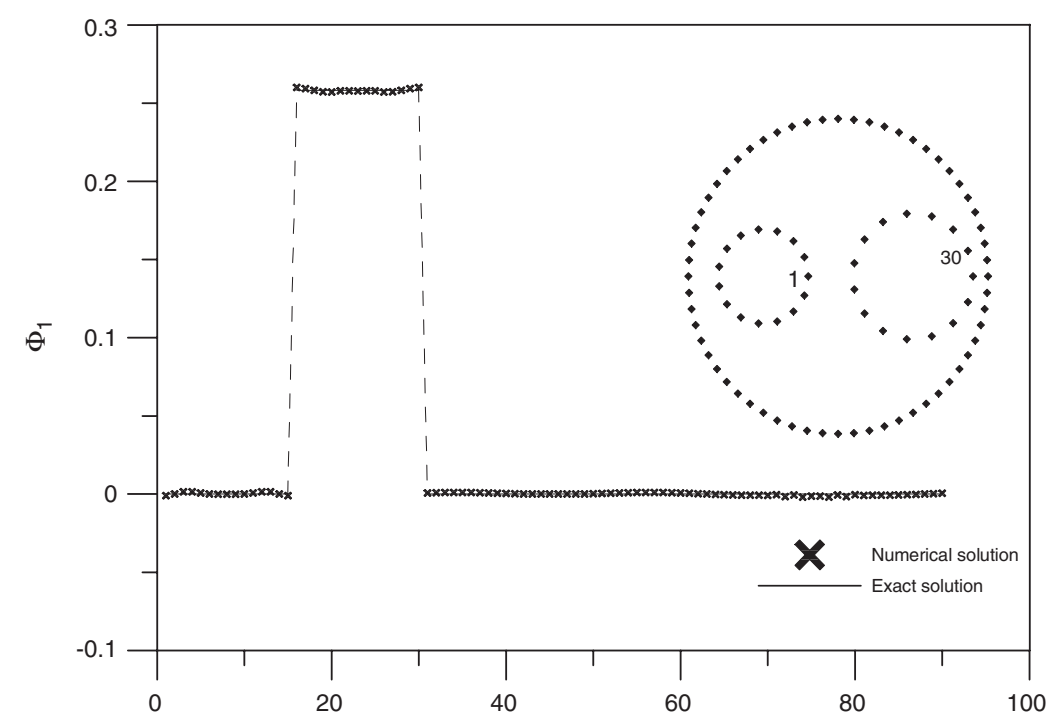

(a)

Element label

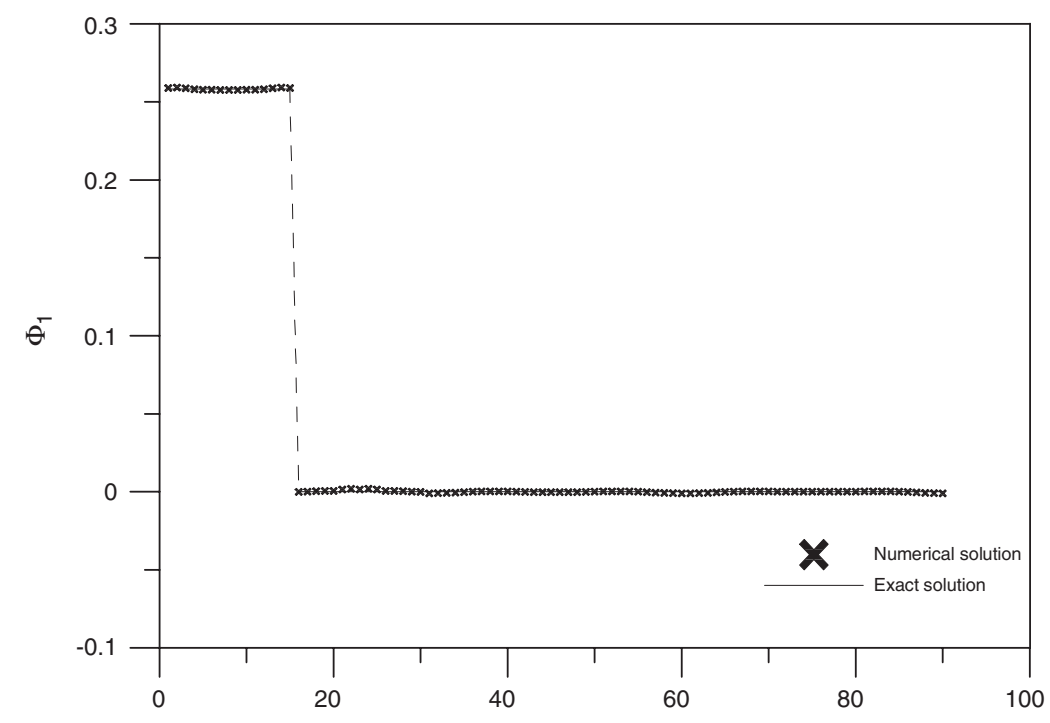

(b)

Element label

Figure 3 . The spurious boundary mode $\Phi_{1}$ along the boundary (1-15, inner left circle; $16-30$, inner right circle; 31-90, outer boundary): (a) $k=6.16\left(J_{0}^{1} / c_{2}=6.012\right)$; (b) $k=8.20\left(J_{0}^{1} / c_{3}=8.016\right)$; (c) $k=9.65\left(J_{1}^{1} / c_{2}=9.579\right)$; and (d) $k=13.00\left(J_{1}^{1} c_{3}=12.772\right)$. 

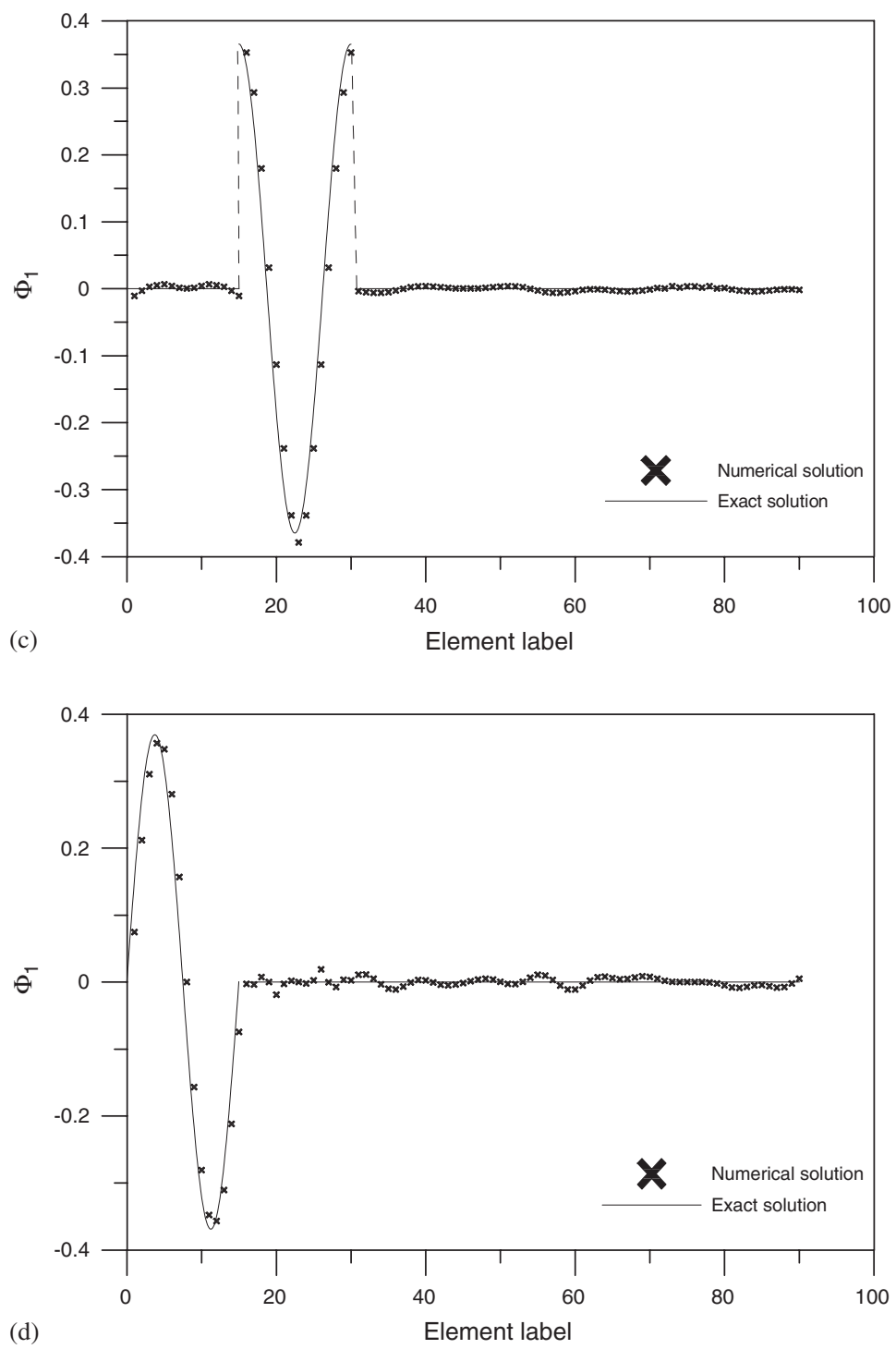

Figure 3. Continued.

in Figure 4, good agreement is obtained. Although the mode shapes corresponding to the eigenvalues $k_{3}$ and $k_{4}$ seem different between the results of BEM and FEM, each mode shape of BEM can be linearly superimposed by using the two independent mode shapes obtained by using the FEM, and vice versa. Similarly, the mode shapes corresponding to the eigenvalue $k_{1}$ are different by a factor -1 between the BEM and the FEM. In this case, the first spurious eigenvalue $k_{\mathrm{s}}=8.196\left(\mathrm{~J}_{0}^{1} / \mathrm{c}=8.016\right)$ is out of the range of $0<k \leqslant 8$; hence, there is no spurious eigenvalue appearing in the range of the former five eigenvalues $\left(k_{1}<k<k_{5}\right)$. For the sake of 
Table II. The former five eigenvalues for a multiply-connected problem with two, four and seven equal holes using different approaches.

\begin{tabular}{|c|c|c|c|c|c|c|c|c|c|}
\hline & PM & BEM & FEM & PM & BEM & FEM & Lin [21] & BEM & FEM \\
\hline$k_{1}$ & 4.548 (SS) & 4.50 (SS) & 4.453 & 4.655 (SS) & 4.47 (SS) & 4.443 & 0.3141 & 7.68 & 7.533 \\
\hline$k_{2}$ & 4.588 (AS) & 4.50 (AS) & 4.512 & NO & 5.37 (AS) & 5.316 & 1.4487 & 8.01 & 7.821 \\
\hline$k_{3}$ & 6.457 (AA) & 6.37 (AA) & 6.267 & NO & 5.37 (SA) & 5.320 & 1.8141 & 8.01 & 7.828 \\
\hline$k_{4}$ & 6.472 (SA) & 6.37 (SA) & 6.269 & $5.561(\mathrm{SA})$ & $5.54(\mathrm{AA})$ & 5.486 & 2.3654 & 8.34 & 8.100 \\
\hline$k_{5}$ & 7.083 (SS) & 7.16 (SS) & 6.930 & 5.868 (SS) & $5.95(\mathrm{SS})$ & 5.884 & 3.2939 & 8.34 & 8.106 \\
\hline
\end{tabular}

demonstrating the singularity pattern resulting in a null-field solution, we plotted the boundary vectors $\Phi_{1}$ and $\Phi_{2}$ from the left unitary matrix of SVD corresponding to the zero singular values for the spurious eigenvalues $8.196\left(J_{0}^{1} / c=8.016\right)$ and $13.06\left(J_{1}^{1} / c=12.772\right)$ as shown in Figure 5(a) and 5(b), respectively, where 15 elements for each inner boundary and 60 elements for the outer boundary are used. Since the radii of inner boundaries are identical to each other, the spurious eigenvalue $k_{\mathrm{s}}=8.196\left(J_{0}^{1} / c=8.016\right)$ has multiplicity of two and the boundary denisties, $\Phi_{1}$ and $\Phi_{2}$, are zero except on the inner boundaries. This is the reason why Figure 5(a) and 5(b) have two boundary modes. From the plot of another spurious eigenvalue $k_{\mathrm{s}}=13.06\left(J_{1}^{1} / c=12.772\right)$, it is similar that the non-zero boundary densities $\Phi_{1}$ and $\Phi_{2}$ appear only in the inner boundaries. In addition, the curve shapes of non-zero boundary densities $\Phi_{1}$ and $\Phi_{2}$ depend on the spurious eigenvalue $k_{\mathrm{s}}=J_{0}^{1} / c$ or $k_{\mathrm{s}}=J_{1}^{1} / c$ with $\mathrm{e}^{\mathrm{i} 0 \theta}$ and $\mathrm{e}^{\mathrm{i} 1 \theta}$, respectively.

\subsection{A circular domain with four equal holes}

The outer boundary with a radius $R=1 \mathrm{~m}$ and four equal circular inner boundaries with radii $c=0.1 \mathrm{~m}$ are considered and the former five eigenvalues are shown in Table II. The four centers of the circular inner boundary locate on the positions of $(0.5,0),(0,0.5),(-0.5,0)$ and $(0,-0.5)$, respectively. Only the Dirichlet boundary condition is considered here. For this problem, Nagaya and Poltorak [22] also used the point-matching approach for finding the eigenvalues. The former five eigenvalues by using the BEM and the FEM are obtained. All the results are listed in Table II including the data of the point-matching method [22]. It is found that the method of point-matching missed the eigenvalues of $k_{2}$ and $k_{3}$ while the $\mathrm{BEM}$ and the FEM obtained. For the eigenvalues of $k_{2}$ and $k_{3}$ or $k_{4}$ and $k_{5}$, the BEM in 

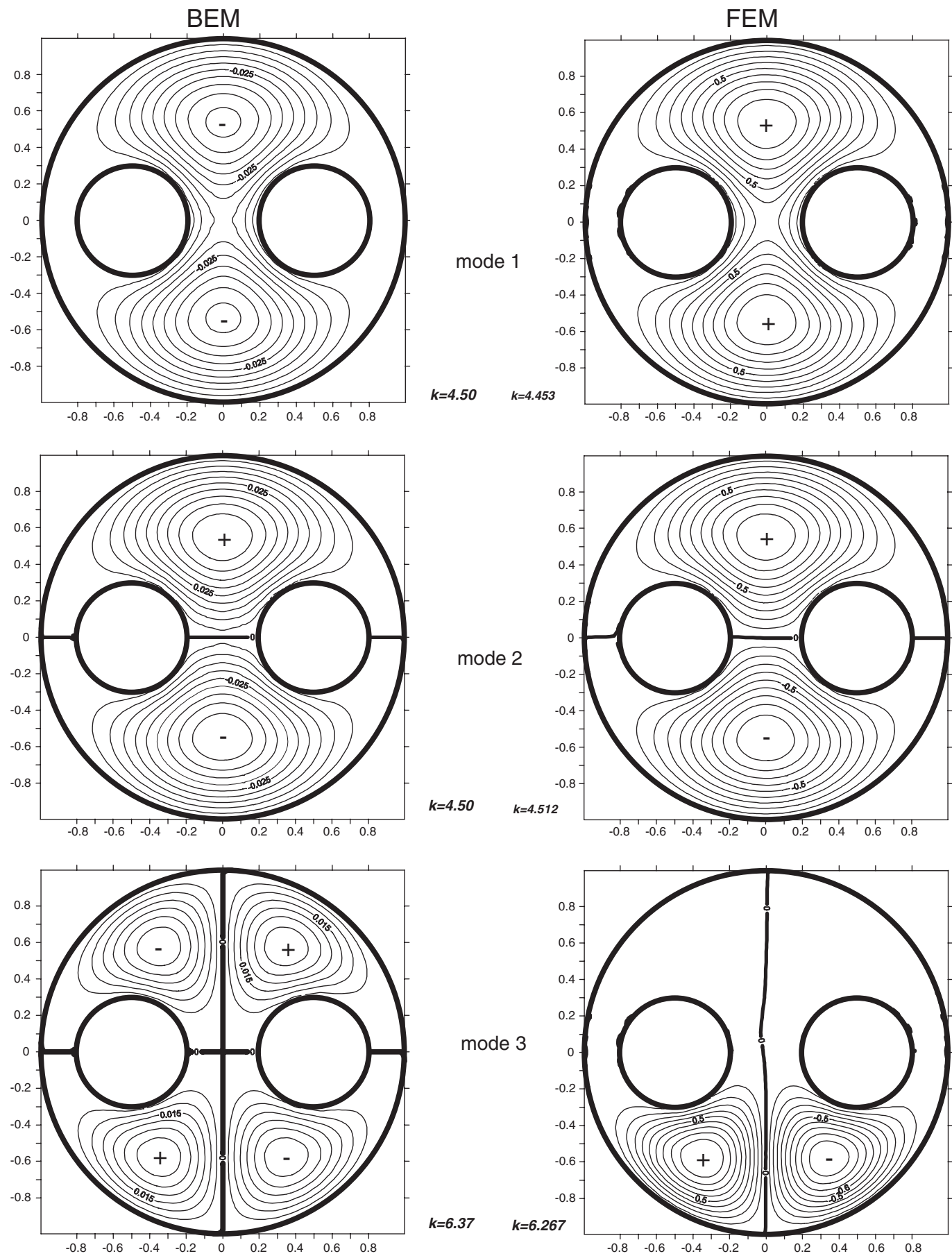

Figure 4. The former five modes for a multiply-connected problem with two equal holes. 

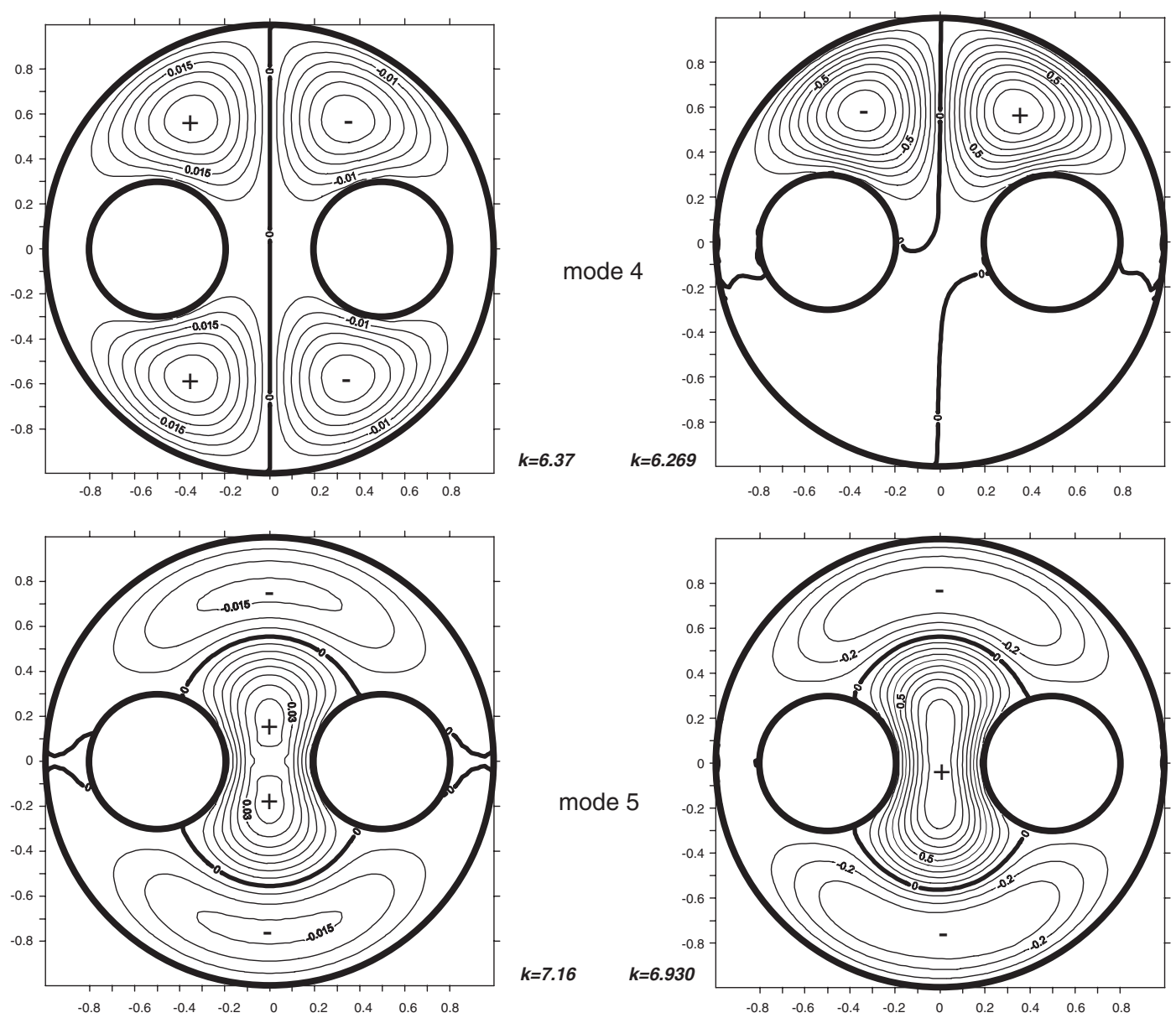

Figure 4. Continued.

conjunction with SVD indicated that they are both roots of multiplicity two by finding the second successive zero singular value in SVD; however, the FEM obtained the two eigenvalues but they are not the same value. Besides, the symmetry of the mode shape predicted by the point-matching method is quite different from that of BEM in $k_{4}$ while the results of FEM match the BEM's data well where the mode shapes are shown in Figure 6. Although the mode shapes corresponding to the eigenvalues $k_{2}$ and $k_{3}$ seem different between the results of BEM and FEM, each mode shape of BEM can be linearly superimposed by using the two independent mode shapes of FEM, and vice versa. Similarly, the mode shapes corresponding to the eigenvalues $k_{4}$ and $k_{5}$ are different by a factor -1 between the BEM and the FEM. Since the minimum spurious eigenvalue $k_{\mathrm{s}}=24.048$ satisfying $J_{0}\left(0.1 k_{\mathrm{s}}\right)=0$ occurs out of the range of $0<k \leqslant 6$, it is consistent that no spurious eigenvalues occurred in the range of $k<k_{5}$. 


\subsection{A circular domain with seven equal holes}

A circular domain of a radius $R=1 \mathrm{~m}$ with seven equal holes of radii $c=0.156 \mathrm{~m}$ is considered and the former five eigenvalues are shown in Table II. Lin [21] used the technique of transformation of cylindrical wave functions to deal with the eigenproblem. The results of Lin's, the BEM and the FEM are shown in Table II. The difference between the BEM's data and the FEM's data is less than 3\%; however, Lin's results for eigenvalues seem to
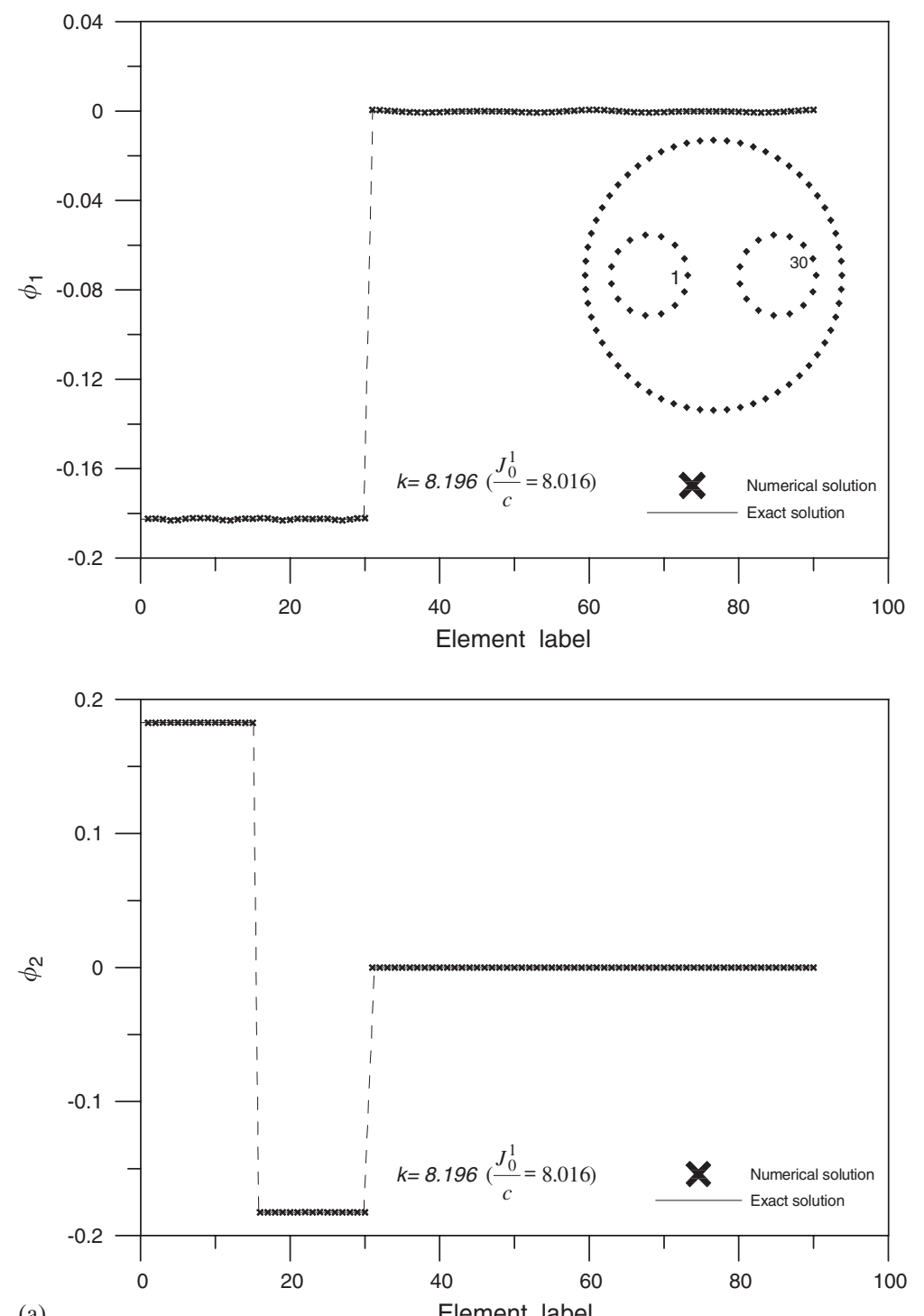

(a)

Figure 5. The spurious boundary modes $\Phi_{1}$ and $\Phi_{2}$ along the boundary (1-15, inner left circle; 16-30, inner right circle; 31-90, outer boundary) $-k=8.196\left(J_{0}^{1} / c=8.016\right)$; and (b) $k=13.06\left(J_{1}^{1} / c=12.772\right)$. 

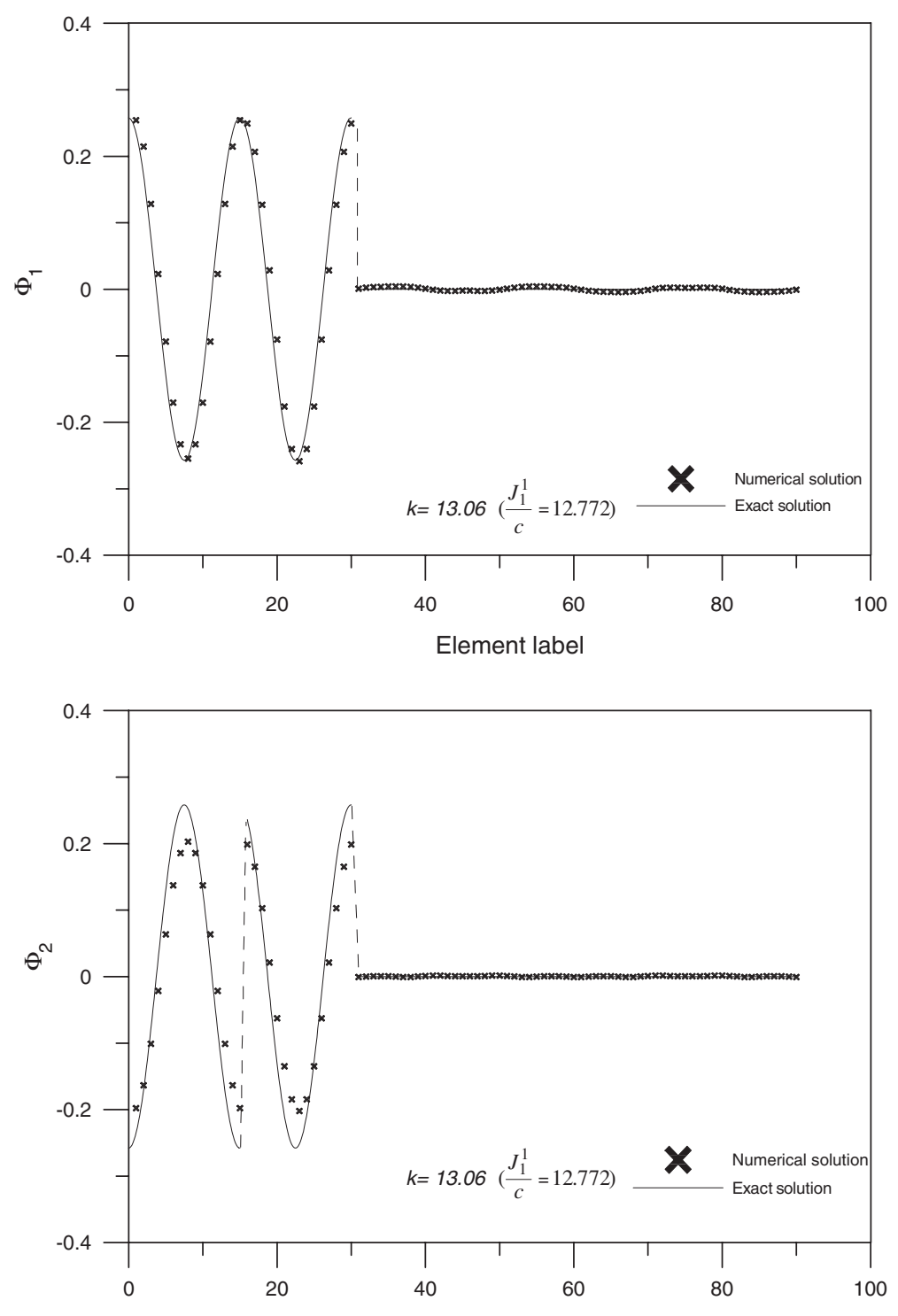

(b)

Element label

Figure 5. Continued.

deviate the results of BEM and FEM. Similarly, FEM cannot identify the root of multiplicity two while the BEM can distinguish by using the SVD technique. Although the mode shapes corresponding to eigenvalues $k_{2}$ and $k_{3}$ or $k_{4}$ and $k_{5}$ seem different between the results of BEM and FEM, each mode shape of BEM can be linearly superimposed by using the two independent mode shapes of FEM as shown in Figure 7, and vice versa. Because the radii of inner boundaries are $0.156 \mathrm{~m}$, the minimum spurious eigenvalue $k_{\mathrm{s}}=15.415$ satisfying 

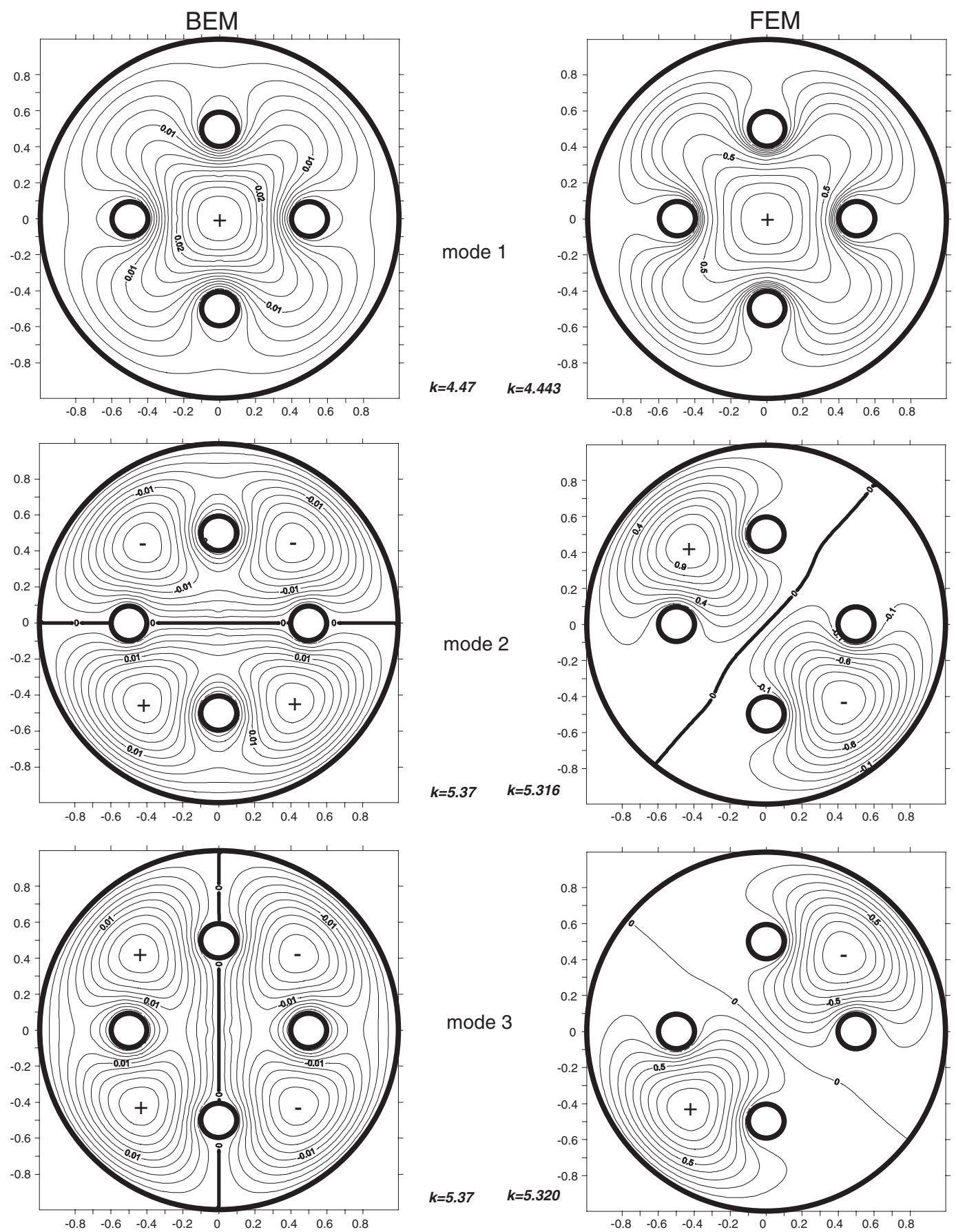

Figure 6. The former five modes of a multiply-connected problem with four equal holes. 

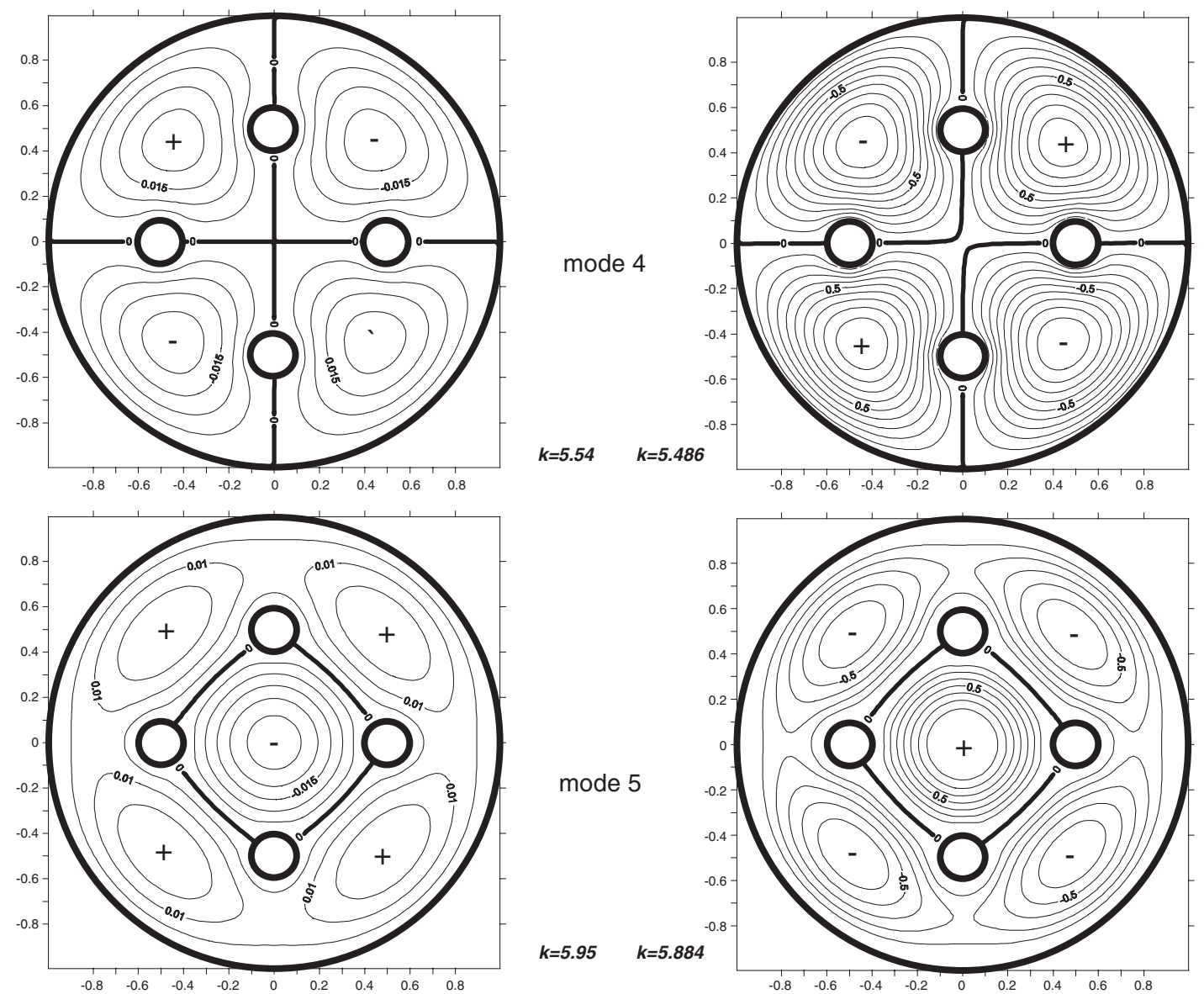

Figure 6. Continued.

$J_{0}\left(0.156 k_{\mathrm{s}}\right)=0$ occurs out of the range of $0<k \leqslant 9$. No spurious eigenvalues occurred in the range of $k<k_{5}$.

\section{CONCLUSIONS}

In this paper, the SVD updating techniques and the Fredholm alternative theorem were employed to deal with the problem of spurious eigenvalue occurring in the truly mutiplyconnected problems. For the direct BEM, the SVD updating documents in conjunction with the Fredholm alternative theorem were ulitized to detect spurious eigenvalues while the SVD updating terms were employed to filter out the true eigenvalues. For the indirect BEM, the spurious eigenvalues were detected by using the SVD updating terms and the true eigenvalues were sorted out by using the SVD updating documents in conjunction with the Fredholm alternative theorem. Spurious eigensolutions are found to be dependent on the formulation while true eigensolutions depend on the types of boundary condition. The numerical experiments 

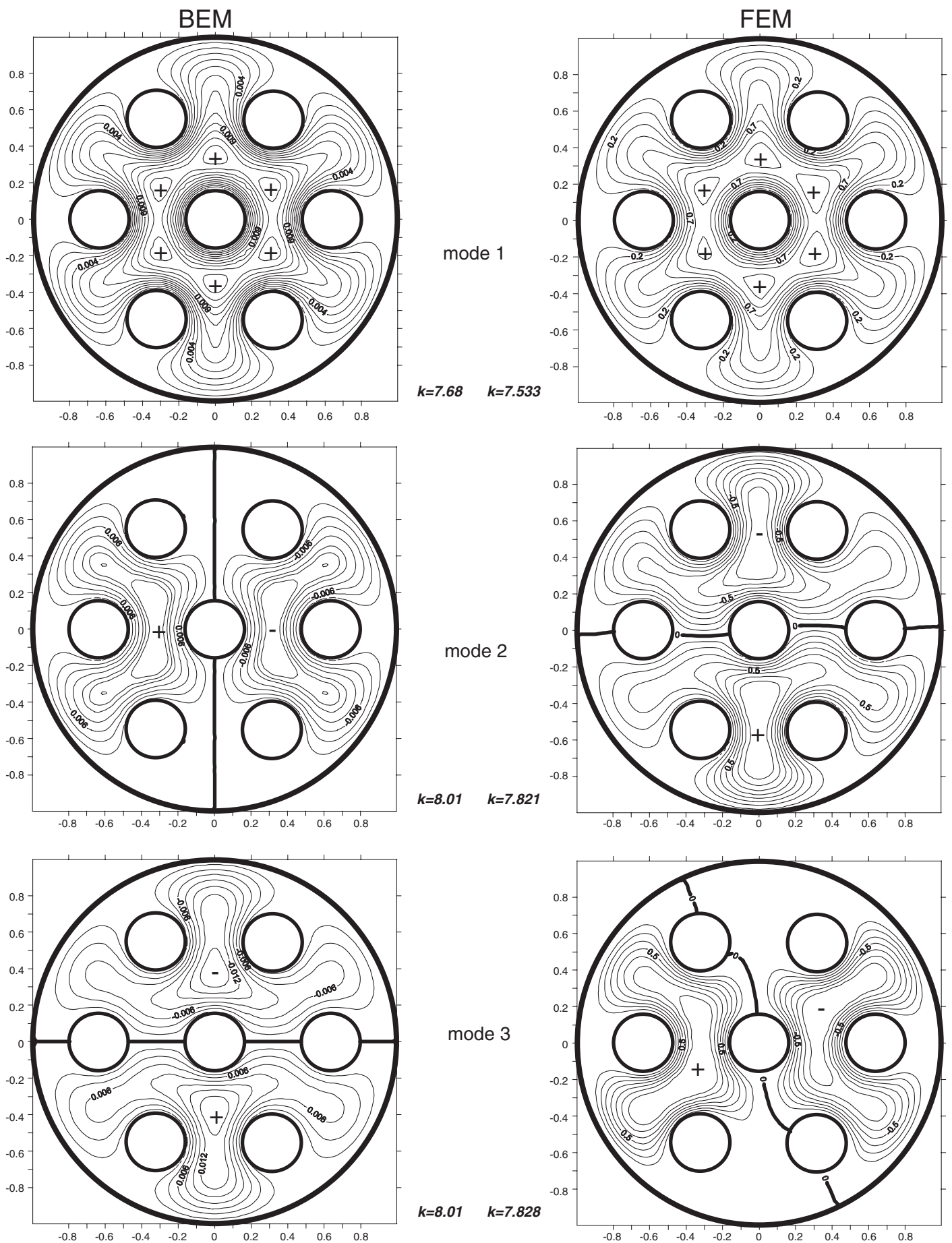

Figure 7. The former five modes of a multiply-connected problem with seven equal holes. 

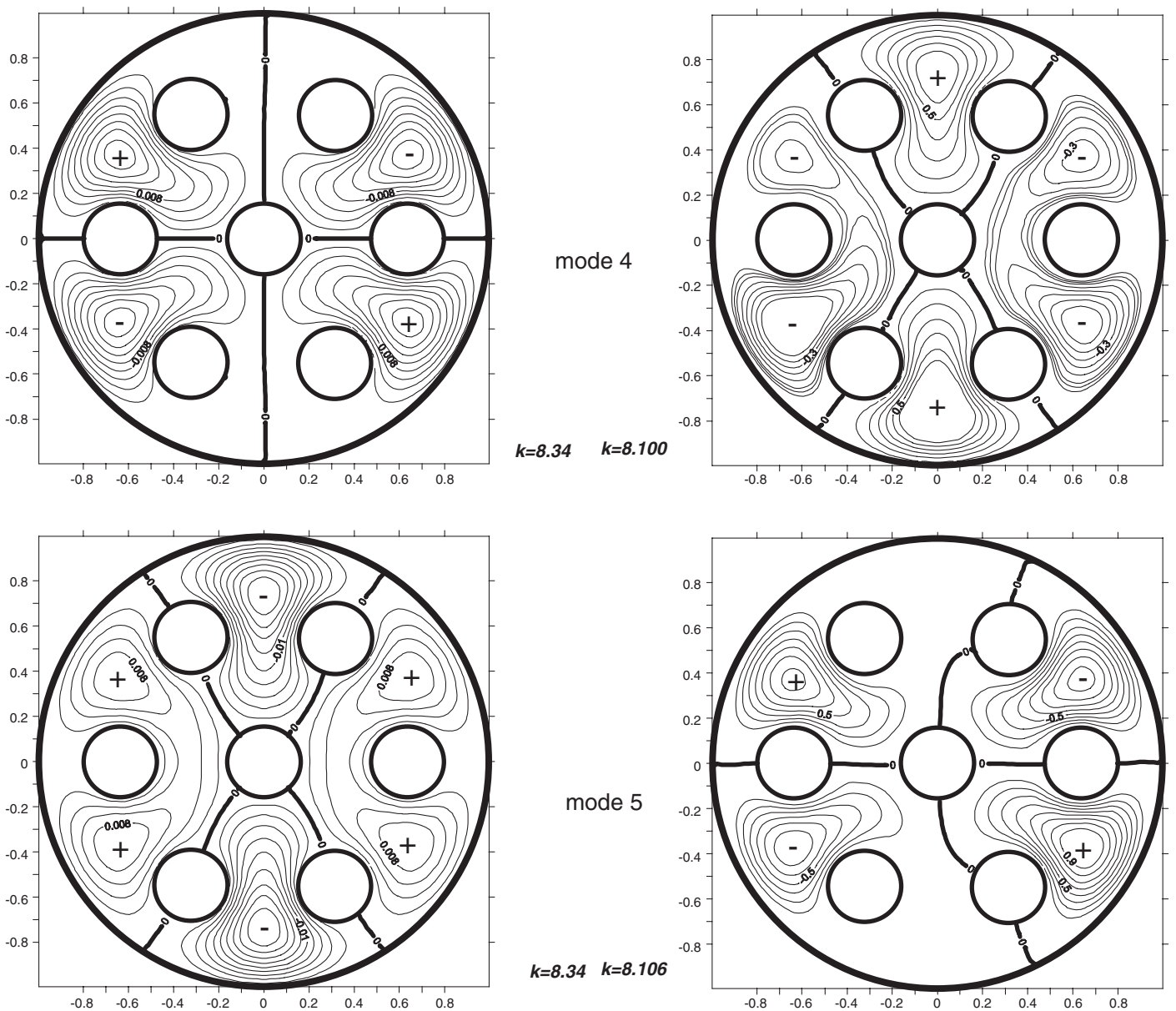

Figure 7. Continued.

of the multiply-connected problems were performed to demonstrate the validity of the arguments which we have proposed above. It was found that the occurring mechanism of spurious eigenvalue depended on inner boundaries. Good agreement between the results of BEM and FEM was made. In addition, the ability of detecting the root of multiplicity two can be achieved in the BEM by using the SVD techniques.

\section{ACKNOWLEDGEMENTS}

Financial support from the National Science Council, under Grant No. NSC-91-2211-E-019-009 and Wu Ta-You Award to National Taiwan Ocean University are gratefully acknowledged.

\section{REFERENCES}

1. Partridge PW, Brebbia CA, Wrobel LC. The Dual Reciprocity Boundary Element Method. Computational Mechanics Publications: Southampton, 1992. 
2. Nowak AJ, Neves AC (eds). Multiple Reciprocity Boundary Element Method. Computational Mechanics Publications: Southampton, 1994.

3. Itagaki M, Nishiyama S, Tomioka S, Enoto T, Sahashi N. Power iterative multiple reciprocity boundary element method for solving three-dimensional Helmholtz equation. Engineering Analysis with Boundary Elements 1997; 20:113-121.

4. Kamiya N, Andoh E, Nogae K. A new complex-valued formulation and eigenvalue analysis of the Helmholtz equation by boundary element method. Advances in Engineering Software 1996; 26:219-227.

5. Tai GRG, Shaw RP. Helmholtz equation eigenvalues and eigenmodes for arbitrary domains. Journal of the Acoustical Society of America 1974; 56:796-804.

6. De Mey G. Calculation of the Helmholtz equation by an integral equation. International Journal for Numerical Methods in Engineering 1976; 10:59-66.

7. De Mey G. A simplified integral equation method for the calculation of the eigenvalues of Helmholtz equation. International Journal for Numerical Methods in Engineering 1977; 11:1340-1342.

8. Hutchinson JR. An alternative BEM formulation applied to membrane vibrations. In Boundary Elements VII, Brebbia CA, Maier G (ed.). Springer: Berlin, 1985.

9. Chen JT, Huang CX, Wong FC. Determination of spurious eigenvalues and multiplicities of true eigenvalues in the dual multiple reciprocity method using the singular value decomposition technique. Journal of Sound and Vibration 2000; 230:219-230.

10. Kuo SR, Chen JT, Huang CX. Analytical study and numerical experiments for true and spurious eigensolutions of a circular cavity using the real-part dual BEM. International Journal for Numerical Methods in Engineering 2000; 48:1404-1422.

11. Berry MW, Drmac Z, Jessup ER. Matrices, vector spaces, and information retrieval. SIAM Review 1999; 41: $335-362$.

12. Golub GH, Van Loan CF. Matrix Computations (2nd edn). The Johns Hopkins University Press: Baltimore, 1989.

13. Kuo SR, Yeih W, Wu YC. Applications of the generalized singular-value decomposition method on the eigenproblem using the incomplete boundary element formulation. Journal of Sound and Vibration 2000; 235:813-845.

14. Chen IL, Chen JT, Kuo SR, Liang MT. A new method for true and spurious eigensolutions of arbitrary cavities using the CHEEF method. Journal of the Acoustical Society of America 2001; 109:982-999.

15. Schenck HA. Improved integral formulation for acoustic radiation problem. Journal of the Acoustical Society of America 1968: 44:41-58.

16. Seybert AF, Rengarajan TK. The use of CHIEF to obtain unique solutions for acoustic radiation using boundary integral equations. Journal of the Acoustical Society of America 1987; 81:1299-1306.

17. Chen JT, Lin JH, Kuo SR, Chyuan SW. Boundary element analysis for the Helmholtz eigenvalue problems with a multiply connected domain. Proceedings of the Royal Society of London Series $A$ 2001; 457:2521-2546.

18. Kitahara M. Boundary Integral Equation Methods in Eigenvalue Problems of Elastodynamics and Thin Plates. Elsevier: Amsterdam, 1985.

19. Burton AJ, Miller GF. The application of integral equation methods to numerical solution of some exterior boundary value problems. Proceedings of the Royal Society of London Series A 1971; 323:201-210.

20. Chen JT, Liu LW, Hong H-K. Spurious and true eigensolutions of Helmholtz BIEs and BEMs for a multiply connected problem. Proceedings of the Royal Society of London Series A 2003; 459:1841-1924.

21. Lin WH. Guided waves in a circular duct containing an assembly of circular cylinders. Journal of Sound and Vibration 1981; 79:463-477.

22. Nagaya K, Poltorak K. Method for solving eigenvalue problems of the Helmholtz equation with a circular outer and a number of eccentric circular inner boundaries. Journal of the Acoustical Society of America 1989; 85: $576-581$.

23. Nagaya K, Yamaguchi T. Method for solving eigenvalue problems of the Helmholtz equation with a arbitrarily shaped outer and a number of eccentric circular inner boundaries. Journal of the Acoustical Society of America 1991; 90:2146-2153.

24. Chen JT, Chen KH. Dual integral formulation for determining the acoustic modes of a two-dimensional cavity with a degenerate boundary. Engineering Analysis with Boundary Elements 1998; 21:105-116.

25. Chen JT, Hong H-K. Review of dual boundary element methods with emphasis on hypersingular integral and divergent series. Applied Mechanics Reviews 1999; 52:17-33.

26. Matrin PA. On the null-field equations for the exterior problems of acoustics. Quarterly Journal of Mechanics and Applied Mathematics 1980; 27:386-396.

27. Chen JT, Kuo SR, Lin JH. Analytical study and numerical experical experiments for degenerate scale problems in the boundary element method for two-dimensional elasticity. International Journal for Numerical Methods in Engineering 2002, 54:1669-1681. 